\title{
Transposition
}

Musique et Sciences Sociales

9| 2021

Musique et sexualité

\section{L'agentivité d'une île : au cœur des Stigmatisés (1918) de Franz Schreker}

The agency of an island: in the heart of Franz Schreker's The Stigmatized (1918)

\section{Aurore Flamion}

\section{OpenEdition}

Journals

Édition électronique

URL : http://journals.openedition.org/transposition/6183

DOI : 10.4000/transposition.6183

ISSN : 2110-6134

Éditeur

CRAL - Centre de recherche sur les arts et le langage

Référence électronique

Aurore Flamion, «L'agentivité d'une île : au cœur des Stigmatisés (1918) de Franz Schreker »,

Transposition [En ligne], 9 | 2021, mis en ligne le 30 mars 2021, consulté le 23 avril 2021. URL : http:// journals.openedition.org/transposition/6183; DOI : https://doi.org/10.4000/transposition.6183

Ce document a été généré automatiquement le 23 avril 2021.

La revue Transposition est mise à disposition selon les termes de la Licence Creative Commons Attribution - Partage dans les Mêmes Conditions 4.0 International. 


\section{L'agentivité d'une île : au cœur des Stigmatisés (1918) de Franz Schreker}

The agency of an island: in the heart of Franz Schreker's The Stigmatized (1918)

\section{Aurore Flamion}

1 «On s'imaginait une sorte de déferlement gigantesque, une chose démesurée, effrayante aussi, comme le mot de "naturalisme" et le nom même de l'auteur le

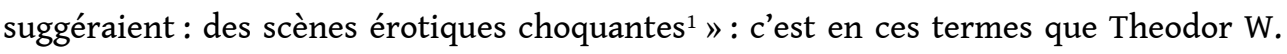
Adorno évoque la création de Die Gezeichneten («Les Stigmatisés ») en 1918 à Francfort à laquelle il assiste, alors jeune adolescent. Ces propos, issus d'une émission de radio produite en $1959^{2}$ rendent compte de la prégnance des fantasmes entourant l'un des opéras les plus connus du compositeur Franz Schreker (1878-1934); rapidement cependant, le musicologue et philosophe reconnaît que la réalité musicale était bien en dessous de ses espérances. L'anecdote est symptomatique de la manière dont s'est constituée la réception de Schreker; les musicologues qui se sont intéressés à l'inscription contemporaine du compositeur dans l'histoire de la musique ${ }^{3}$ font état du même constat. Peu envisagée pour elle-même, constamment considérée comme tributaire d'esthétiques lui préexistant ${ }^{4}$, écrasée par les ombres paradoxalement tutélaires de Debussy et de Wagner ou par les discours nationalistes et antisémites, son œuvre demeure aujourd'hui encore très méconnue; Schreker était pourtant l'un des compositeurs les plus joués en Europe - principalement en Allemagne et en Autriche de l'entre-deux-guerres.

2 Il est saisissant de constater dans le brouillement de ces discours combien l'œuvre en elle-même est négligée. Die Gezeichneten met en scène la tragédie d'Alviano Salvago, un noble caractérisé par sa laideur, et projetant de donner à la ville une île - l'Élysée - qu'il a fait construire pour célébrer la beauté, mais qui en réalité accueille les orgies de la noblesse auxquelles il ne prend pas part ( $\mathrm{I}^{\mathrm{er}}$ acte). Le II $\mathrm{ecte}$ confronte Alviano à une jeune peintre issue de la bourgeoisie, Carlotta, qui le convainc de se laisser peindre ; il en tombe éperdument amoureux. Le III ${ }^{e}$ acte signe la venue de tous les Génois sur l'île ; 
médusés par les scènes auxquelles ils assistent, ils portent aux nues Alviano, bientôt accusé par les notables de la ville des crimes sexuels survenus sur l'île.

Alviano est quant à lui entièrement accaparé par la recherche de Carlotta, dont des éclats de voix fictifs le poursuivent. Carlotta, ivre des beautés de l'île, se livre pendant ce temps à Tamare, le jeune noble orgueilleux qu'elle avait éconduit à plusieurs reprises dans le premier acte. Leur union lui est fatale, comme elle l'avait elle-même prédit au cours du II acte ; sur le point de mourir, elle est évanouie quand Alviano la rejoint. Fou de douleur, excédé par les provocations de Tamare, Alviano le tue; Carlotta, réveillée par le hurlement de Tamare, maudit Alviano et meurt. Ce dernier quitte la scène, ayant perdu toute raison.

4 L'histoire, tragique, se passe au xvI siècle et s'inspire d'un conte cruel d'Oscar Wilde (1854-1900) $)^{5}$, The Birthday of the Infanta (« L'Anniversaire de l'infante »). Elle est cruelle : lors de son anniversaire, une jeune infante fait croire au nain hideux qui lui est offert en cadeau qu'elle éprouve des sentiments pour lui ; le nain s'éprend éperdument d'elle, avant de découvrir la vérité et de mourir de douleur. Ce canevas avait d'abord retenu l'attention d'Alexander von Zemlinsky (1871-1942), qui avait demandé à Schreker d'écrire pour lui un livret à partir du conte. Ce sera fait entre 1911 et 1915, mais ce dernier préférera finalement le garder pour lui-même, et c'est Georg C. Klaren qui fournira le livret tant attendu à Zemlinsky, dont Der Zwerg (« Le Nain») voit le jour en 1922, quatre ans après la création des Stigmatisés.

5 Si l'opéra de Zemlinsky reste assez proche du conte de Wilde, Schreker opère un certain nombre de déplacements importants; le nain - Alviano - est ainsi doté d'une réelle stature sociale, déterminante dans l'économie du livret puisqu'elle lui permet de faire don de l'île à la ville de Gênes. Il n'ignore par ailleurs rien de sa laideur: c'est précisément cette conscience douloureuse qui le pousse à construire l'Élysée, île de tous les fantasmes et temple de la beauté classique. Cela dit, les sources d'inspiration de Schreker ne semblent pas se réduire à L'Anniversaire de l'infante: Alviano est également un lointain descendant du Nain de Schubert ${ }^{6}$, ou de Rigoletto de Verdi ${ }^{7}$. Le III ${ }^{e}$ acte, marqué par la figure érotique d'un faune musicien - il «souffle dans sa syrinx $»^{8}-$, témoigne également d'une proximité avec le Prélude à l'après-midi d'un faune de Debussy. Il n'est néanmoins pas sûr que Schreker ait eu accès à cette œuvre ; Christopher Hailey rappelle ainsi :

On est tenté de postuler l'influence de l'impressionnisme français mais, à part Louise de Charpentier, le compositeur ne connaissait guère la musique française contemporaine à cette époque. Schreker semble émerger de nulle part avec un langage musical tout à fait idiosyncratique, dont l'originalité réside moins dans une fusion d'impulsions que dans un montage audacieux de sources souvent clairement identifiables. ${ }^{9}$

Or, si cet opéra n'a eu cesse de susciter des discours fustigeant l'omniprésence d'une sexualité débridée et délétère ${ }^{10}$, s'attachant indistinctement au texte du livret, à l'esthétique de Schreker, voire à la biographie du compositeur lui-même, ils semblent ne jamais avoir été l'objet d'une analyse circonscrivant et expliquant ce qui, dans l'optique des auteurs concernés, fait le caractère pathologique de l'œuvre. Se contentant de généralités, les commentaires ne semblent pas s'être intéressés à ce qui fonde pourtant un des principaux intérêts de l'opéra: la construction ex nihilo d'un formidable espace dramatique, l'Élysée. Peuplée de faunes, de bacchantes et de nymphes, dédiée à la volupté et aux orgies des Génois, l'île, véritable défi à la mise en scène, décuple les pulsions sexuelles des personnages, jusque-là inhibées. Écrasante 
d'excès et de désir, métaphore des instincts réveillés par les fascinantes «mixtures schrekériennes " ${ }^{11}$, l'île est dotée d'une agentivité propre.

7 Nous définissons le concept d'agentivité appliqué à une œuvre musicale en nous appuyant sur les travaux d'Alfred Gell ${ }^{11}$, en anthropologie de l'art, et ceux du musicologue Robert $\mathrm{S}$. Hatten ${ }^{12}$. Selon ce dernier, relèvent de l'agentivité les configurations musicales capables de « simuler les actions, les émotions et les réactions d'un agent humain $»^{13}$, qui ne sont "pas seulement des projections subjectives mais peuvent être des inférences intersubjectives (partagées) stylistiquement et stratégiquement justifiées par les caractéristiques spécifiques d'une œuvre musicale $»^{14}$, et existent indépendamment des intentions du compositeur ou des interprètes ${ }^{15}$. Tout se passe ainsi, dans Les Stigmatisés, comme si l'élysée, loin de se réduire à un simple espace géographique, se comportait comme une entité toute puissante capable de susciter des comportements irrationnels chez les personnages de l'opéra.

8 S'impose dès lors la question des modalités de sa représentation dans l'économie de l'œuvre: comment Schreker procède-t-il pour créer cet objet insaisissable se nourrissant des ressources illimitées de l'opéra? Avec quelles méthodologies analyser ses mécanismes intradiégétiques? Comment s'organise picturalement, dramaturgiquement, musicalement à l'intérieur de l'œuvre ce que Georges Bataille appelle la «perte [de soi] volontaire impliquée dans l'érotisme $»^{16}$ qui, fondée sur « la fascination pour l'instant qui précède immédiatement la ruine [et] la destruction ${ }^{17}$, pousse les différents protagonistes à abdiquer toute volonté pour rejoindre le chant extatique de l'île?

9 Tenter de saisir la singularité de cet opéra, en accordant une attention égale aux images qu'il convoque et aux complexes sonores inédits qu'il déploie, constitue ainsi le principal objectif de cette étude fondée sur le constat que la dimension sensuelle et érotique, si elle a fait couler beaucoup d'encre, n'a pas fait l'objet d'analyses spécifiques $^{18}$.

\section{Une énergétique intradiégétique}

10 Parler d'agentivité peut sembler quelque peu surprenant pour évoquer un pur objet dramaturgique: comment prêter une quelconque intentionnalité à un espace géographique ? L'île n'est pas l'un des personnages du drame; elle n'est pas davantage l'addition des différentes créatures fantastiques rencontrées au cours du III ${ }^{e}$ acte. Le livret ne recourt pas davantage à l'anthropomorphisme pour la doter d'une volonté individuelle susceptible de produire des effets réels sur les personnages. Et pourtant, force est de constater que des mécanismes inédits dans l'économie de l'œuvre opèrent dès le prélude du III acte, et qu'ils sont directement liés à la venue sur l'île. Ces mécanismes produisent immédiatement des effets singuliers sur le public intradiégétique de l'œuvre, décuplant les instincts sexuels jusque-là refoulés des personnages ${ }^{19}$. Le peuple ${ }^{20}$ est en effet, dès les premières mesures, désarmé par ce qu'il est lui donné à voir : fasciné, partagé entre déférence et inquiétude, l'Élysée lui paraît être un nouvel Éden. L'échange entre le Premier Bourgeois et son fils, au tout début de la première scène, est à cet égard éloquent :

LE PREMIER BOURGEOIS

S'il n'y avait pas ces esprits dissolus

Je dirais : c'est le paradis. 


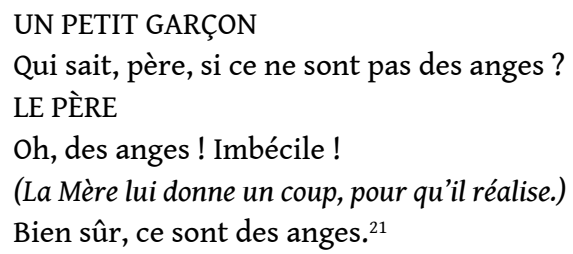
tenter de cerner maladroitement la réalité fantastique qui leur est donnée à voir. Inconsistant, le peuple n'oppose pas de réelle résistance aux charmes qui le gagnent progressivement. aussi dans les voluptés de l'île ; elle change radicalement d'attitude dès les premières scènes du III ${ }^{e}$ acte. Inquiète, elle fait part au duc Adorno des sensations troublantes qui la gagnent :

\section{CARLOTTA}

Comme si j'avais bu du vin,

Capiteux et mêlé de racines de sorcière,

Ou ce philtre étrange brassé avec le sang

Du cœur d'une vierge folle d'amour!

C'est le charme terrible de la beauté,

Habituellement prisonnier, à moitié caché aux sens,

Et maintenant déchaîné, livré au jour

Et aux regards désirants. ${ }^{22}$ quelques scènes plus loin à Tamare, le jeune noble violent qu'elle avait vigoureusement repoussé dans les deux premiers actes. La femme courageuse et déterminée des deux premiers actes semble subitement se dissoudre dès son arrivée sur l'île : inconstante, elle fuit Alviano - elle lui avait pourtant déclaré ses sentiments lors de la scène de l'atelier, acte II scène II - et parait avoir tout oublié de sa vie passée. En ce sens elle apparaît comme une véritable "femme weiningérienne $"^{24}$, d'après Otto Weininger (1880-1903), l'auteur de l'essai antisémite et antiféministe, Sexe et caractère, abondamment lu au début $\mathrm{du} \mathrm{xx}^{\mathrm{e}}$ siècle, qui fait de la femme, dépourvue d'essence, réduite à la sensation pure, l'incarnation de la tentation, du péché et de l'abandon à une sexualité sans entraves. Dans l'opéra, Carlotta est désormais réduite aux instincts qui la submergent progressivement. Dès lors, selon la musicologue Sherry D. Lee, « l'abandon total à la sexualité l'emporte non seulement sur la rationalité et la moralité, mais aussi sur de nobles idéaux intellectuels ou créateurs $»^{25}$. Le message semble clair: l'anéantissement figuré de la jeune femme ${ }^{26}$ annonce son anéantissement littéral, et dessine la trajectoire à laquelle semblent promis les visiteurs de l'île. Alviano, les Sénateurs et le duc Adorno échappent quant à eux miraculeusement aux séductions de l'île : cette inflexibilité était nécessaire, du côté des garants de l'ordre et de la morale, pour pouvoir faire entendre au sein de l'œuvre des discours pathologisants sur les licences sexuelles des Génois. Elle s'explique, dans le cas d'Alviano, par l'inquiétude de perdre Carlotta qui le presse et qui agit comme un puissant antidote ${ }^{27}$.

\section{La fascination érotique de la stase}

14 Évoquer les effets produits par l'île sur les Génois dit peu des mécanismes à l'œuvre sur l'Élysée. Alain Leduc, dans son article consacré aux Stigmatisés, considère que l'Élysée 
est en réalité le fruit d'un «fantasme compensatoire » d'Alviano, et de sa "projection narcissique "; selon lui, il s'agit de compenser les défauts de son apparence physique, le personnage étant petit et contrefait ${ }^{28}$. Aussi séduisante que puisse être cette hypothèse, elle nous semble insuffisante pour tenter d'approcher ce qu'il se joue d'un point de vue dramaturgique. La théorie d'une agentivité de lî̂le, en effet, ne peut se réduire à l'analyse psychologique des personnages déconnectant le livret des dispositifs scéniques mis en œuvre par Schreker, ou des ressources musicales déployées au même moment. Ces dispositifs scéniques et musicaux reposent sur une ambition, placer le spectateur - intradiégétique, ou extradiégétique - dans une situation de fascination non médiatisée par le langage verbal, mais entièrement prise en charge par la sidération de la vue - le tableau vivant - et de l'audition - le Klang. L'Élysée ne se résume ni au « corps séduisant qu'Alviano aurait aimé posséder ", ni à un «Disneyland pour adultes ${ }^{29}$ : elle constitue bien plutôt une tentative, sur deux plans différents, de déstabiliser le spectateur (intra ou extradiégétique). Arnaud Rykner, dans son ouvrage Corps obscènes, rappelle la puissance obstinée de certaines images qui «semblent s'être donné le mot pour inquiéter le discours, et, le faisant trembler, vaciller sur ses bases, l'obligeant à lâcher prise, à se repenser non plus comme vecteur de transmission du monde mais comme écran entre le monde et nous ${ }^{30}$. Le tableau inaugural peint par la didascalie du $\mathrm{III}^{\mathrm{e}}$ acte est de celles-là : particulièrement longue, elle constitue une hypotypose proche, pour les protagonistes, du tableau vivant. Immobilité, intensité subite de l'image, ambiance néo-classique, recours à l'antique, importance de la statuaire grecque classique: autant d'éléments partagés avec cette forme d'art qui désigne pour Bernard Vouilloux «en un sens élargi et, en quelque sorte, transhistorique, [...] le moment où le corps, sans nécessairement faire référence à un tableau ou à une sculpture déterminés, fait tableau $» .^{31}$

\section{L'ekphrasis comme subjugation visuelle}

Quel que soit le regard porté sur l'île, cette dernière semble inlassablement échapper à toute rationalisation de son agentivité. Dotée d'un pouvoir agissant nécessitant une véritable "phénoménologie de l'image active ${ }^{32}$, l'Élysée, dans son immanence, renouvelle le modèle de Pygmalion, qui pour Carole Halimi, constituait dans la littérature du XIX ${ }^{e}$ siècle le « modèle de l'œuvre agissante, jusqu'à en faire une créature dont la vie dévore celle du modèle, du créateur, parfois les deux " ${ }^{33}$. L'Élysée est né du désir d'Alviano, évoqué à plusieurs reprises lors de l'opéra ${ }^{34}$; mais l'île, telle qu'elle apparaît au début du III ${ }^{\mathrm{e}}$ acte, est désormais autonome, et produit ses propres effets sur les différents personnages de l'opéra. Extrêmement longue, la didascalie initiale place d'emblée l'acte final sous le sceau de l'ekphrasis. Convoquant archaïsme, paganisme et fantastique, le paysage décrit ici est envisagé comme le « relais visuel et agissant d'une sensation magique ", fondé sur la création d'un "acte d'image [qui] se joue dans l'intensité de cette immobilité $»^{35}$.

L'île appelée Élysée. La scène donne l'impression d'un jardin paradisiaque. Tout à fait au fond, à gauche des spectateurs, on devine les contours de la ville de Gênes et la mer qui brille dans la lumière du crépuscule. À droite des spectateurs, la scène s'élève en pente douce; vers le fond, la scène s'élève en paysage rocheux. Un chemin conduit à un endroit où l'on voit un épais buisson de roses aux couleurs saturées. Le milieu de la scène presque jusqu'au fond est couvert d'herbe verte, de fougères arborescentes et de fleurs, principalement de couleurs vives. Dans les 
bosquets touffus, scintillent de petits kiosques aux formes fantastiques et des groupes de marbre, qui représentent essentiellement des scènes érotiques de la mythologie grecque. De hautes gerbes d'eau surgissent de fontaines s'éclairant à la tombée de la nuit. Çà et là, la silhouette d'un faune surgit d'un bosquet, comme si elle donnait vie au marbre. Des groupes de naïades passent, un cortège de bacchantes se presse de côté, poussant des cris aigus. Les premiers visiteurs se montrent. Muets d'étonnement, ils se promènent parmi ces merveilles. On entend au loin, venant de la ville, les cloches de l'angélus. Pendant l'angélus, les apparitions païennes disparaissent, les bourgeois s'agenouillent et se découvrent. Entre-temps, la nuit s'est faite. De la ville au loin parvient un rai brumeux de lumière rouge sombre. La surface de la mer est comme parsemée de milliers de petites lumières : les petits bateaux des invités de la fête s'approchent. Les fontaines commencent à s'éclairer. Ici et là, dans les bosquets, des flammèches scintillent, telles des lucioles. Une ombrelle de fleur jette une lueur verte sur un groupe sculpté représentant un couple s'étreignant avec ardeur. La canaille païenne s'enhardit et un petit elfe inonde de fleurs un honorable bourgeois génois. Un faune musicien sur la scène. Attirés, d'autres faunes s'approchent, se rassemblent autour du faune qui souffle dans sa syrinx. Un cortège de naïades passe, les faunes les suivent. Musique venant de la ville; le faune écoute. Les naïades s'enfuient dans des directions opposées, pourchassées par les faunes. Étonnement craintif des gens qui arrivent timidement par petits groupes. Un faune attrape une naïade. Scène entre les deux : il la courtise, elle se défend, il la presse, à la fin elle se donne. Le jeu va crescendo jusqu'à une passion sauvage. Un cortège de bacchantes se déchaîne à l'arrière-plan avec des cris perçants ; puis bienheureux épuisement.

Retour des faunes et des naïades en couples. Jeux amoureux, on se couche dans l'herbe et les bosquets sur les côtés. Les premiers bourgeois se montrent, le peuple de la ville. Le faune joue à nouveau. Ambiance du soir. Bruits de cloches venant de la ville (angélus) à peine perceptible. Les bourgeois s'agenouillent, se découvrent. Les silhouettes païennes se retirent pendant la durée de l'angélus, puis reviennent. Jeu discret avec les citoyens qui, à quelques exceptions près, restent sur la défensive et se signent à plusieurs reprises. Ce jeu se poursuit discrètement jusqu'au début de la scène II environ. Par la suite, plus rien ne devra détourner l'attention. Seuls un faune ou une naïade curieux, ici et là, épieront depuis quelque buisson ou derrière une colonne. Au fond, des gens du peuple traversent la scène en se promenant.

Les Génois eux-mêmes ont conscience de la stase que constitue cette scène, au sens d'une fixation dans le temps d'une action interrompue. À ce propos, il n'est pas anodin qu'ils comparent spontanément le spectacle auquel ils assistent au régime pictural du tableau ${ }^{36}$, ou insistent sur sa présence incarnée ${ }^{37}$. La didascalie, en effet, n'est pas sans faire penser aux paysages rococos d'Antoine Watteau (Le Festival de l'Amour [1717], Le Pèlerinage à l'île de Cythère [1717]) et de Francesco Zuccarelli (Bacchanale [1740-50]) ${ }^{38}$, célébrant les fêtes galantes de jeunes gens se livrant au plaisir amoureux sous l'égide de faunes, de Cupidons ou d'Aphrodite.

Présentant de manière explicite des scènes sexuelles - viols, masturbation, orgies -, la didascalie obéit par ailleurs à une progression logique, analysée par Alain Leduc dans son article consacré aux Stigmatisés : il s'agit de séduire le public intradiégétique « en le soumettant à différents types d'impressions sensorielles et [en le faisant] passer progressivement d'un état d'excitation sensuelle assez anodine à la lascivité la plus débridée. L'aboutissement implicite de ce parcours initiatique étant la grotte secrète où en toute logique la tension érotique accumulée est appelée à se libérer dans l'acte sexuel $»^{39}$.

18 La force du tableau ne se résume néanmoins pas aux actions qu'il met en scène, même très explicites; les images qu'il impose, parce qu'elles charrient un sens latent, ne 
contribuent pas moins à subjuguer les spectateurs. Dans cette perspective, la figure du faune ne doit rien au hasard, mais contribue au contraire à nourrir la force agissante de l'élysée. Christophe Bataillé rappelle ainsi les conceptions véhiculées par le faune dans l'Antiquité :

On sait combien la Nature apparaissait aux Anciens comme un lieu animé, toute chose, aussi minérale soit-elle, se trouvant habitée par le vivant, douée d'une vie intrinsèque. Il n'y a pas jusqu'au langage lui-même qui ne soit comme contaminé par cette conception panthéiste du monde ${ }^{40}$.

19 Le choix même de la période historique exacerbe l'érotisme de la scène. Si Schreker écrit livret et musique pour un public qui ne verra l'œuvre que dans l'entre-deuxguerres, ce sont deux autres périodes, fantasmées, qui en constituent le cadre temporel : la Renaissance et l'Antiquité, la première enchâssant la seconde dans le III ${ }^{\mathrm{e}}$ acte. L'Antiquité est le signe d'un hédonisme sans entraves, dont l'impudeur suscite l'inhibition et la crainte des personnages qui « restent sur la défensive et se signent à plusieurs reprises». Fréquemment associée aux orgies qu'elle peut susciter ${ }^{41}$, cette période fantasmée de l'histoire est également «le symbole d'une existence où la satisfaction des désirs l'emporte sur les contraintes morales étouffantes et comme l'adversaire déclaré dans le même temps d'un christianisme qui tend à culpabiliser et donc à inhiber toute la sexualité féminine $»^{42}$.

Deux univers s'affrontent en effet dans cette didascalie : celui, caractérisé par l'excès, des bacchantes, faunes et naïades, et celui des Génois. Chacun possède son espace sonore et visuel : aux «cloches » et à "l'angélus » de la ville, répondent les appels de quinte du cor anglais dès la troisième mesure qui signalent l'apparition du faune sur la scène $\mathrm{e}^{43}$, tout en rappelant par ce geste l'érotisme $\mathrm{e}^{44}$ et l'onanisme attachés à sa figure. Le musicologue Tim Steinke, dans cette perspective, souligne que ce geste expressif - le faune qui souffle dans sa syrinx - rend visible et explicite l'activité des faunes et des naïades, entièrement sexuelle ${ }^{45}$. Aux jeux de ces créatures fantastiques répondent, en contraste, les échos de Gênes que l'on perçoit encore ; «à peine perceptibles », les deux cors placés en coulisse évoquent la réalité rassurante de la ville ("Musik aus der Stadt"), à mesure que le peuple arrive sur l'île. Le reste du prélude est consacré aux saynètes sexuelles et parfois violentes se jouant entre les faunes et les naïades que contemplent, médusés, les Génois.

De la fascination à la dévoration, il n'y a qu'un pas $^{46}$; terrassant littéralement les spectateurs intradiégétiques, fascinante, insaisissable, l'île signe leur dissolution dans une sensualité exacerbée. Proche de l'univers sadien - le parallèle est fait par l'Opéra de Lyon, dans une vidéo de présentation de l'opéra, joué au cours de la saison $2015^{47}$ l'Élysée met en place un véritable espace dramaturgique qui rompt avec le réalisme des deux premiers actes. L'île constitue à cet égard ce qu'Annie Le Brun appelle « espace inobjectif »: " ni subjectif, ni objectif », véritable "espace de jeu, à tous les sens du terme ", il est "l'"aire de l'informe", où objets et représentations mentales, "joints et séparés", peuvent prendre forme $»^{48}$ et perdre les spectateurs dans un abyme insondable.

22 Le cor anglais, suivi du cor de basset, signe la fin de cet épisode fantastique : à l'érotisme du faune qui joue à nouveau sur scène succèdent les cloches de la ville, très lointaines et à peine perceptibles, qui sonnent l'angélus ${ }^{49}$. Les bourgeois se signent et restent sur la défensive, tandis qu'une dernière sonnerie de cor anglais annonce la fin 
du prélude ; altérée et inquiétante - l'appel de quinte s'est transformé en triton -, elle précède la première scène de cet acte, et le retour du Klang schrékerien.

\section{Le Klang schrékerien : incarner musicalement la performativité du désir}

Plus qu'aucun autre paramètre, le son se situe au cœur de l'esthétique de Schreker. Omniprésent dans les articles où Schreker revient sur sa démarche compositionnelle ${ }^{50}$, il constitue un véritable topos dans son œuvre: fasciné par la palette orchestrale ${ }^{51}$, le compositeur fait du son - le Klang - un élément dramaturgique essentiel de ses opéras ${ }^{52}$. À partir de Die ferne Klang ["Le son lointain »] (1912), en passant par Das Spielwerk und die Prinzessin [«Le Carillon et la Princesse»] (1908-1912) et Der Schatzgräber ["Le Chercheur de trésors "] (1918), tous ces opéras thématisent l'attrait mystique, sensuel, dangereux et irrépressible que le son exerce sur les personnages de l'œuvre ${ }^{53}$. Incarnation sensible d'une utopie inaccessible, il "fait signe vers un domaine antéartistique $»^{54}$; principe déstabilisant par excellence, le son [Klang] n'est en effet « lié à aucun système de valeurs établi, qu'il soit musical ou moral », ce dont Paul Bekker, théoricien et grand critique de Schreker avait bien conscience ${ }^{55}$. Plus encore, pour Alain Leduc il est à la musique « ce que la pulsion est à la psyché humaine $»^{56}$. Il connaît enfin dans les Stigmatisés un déploiement inédit : contrairement aux mirages du Son lointain, Schreker ancre ses complexes sonores dans un espace réel intradiégétique, à savoir l'île.

24 Particulièrement dense, le prélude $\mathrm{du} \mathrm{III}^{\mathrm{e}}$ acte en dévoile progressivement les mécanismes dramaturgiques; les mentions de la musique intradiégétique, fondées sur la spatialisation et la fonction signalétique, permettent d'opposer l'univers fantastique de l'Élysée aux échos lointains de la ville de Gènes. Il semble surprenant à cet égard que la didascalie ne fasse pas état d'une transposition, sur le plan musical, des merveilles sonores dont elle est le signe.

En effet, le tableau vivant fantastique et érotique, les mixtures orchestrales du prélude du Ir $^{\text {er }}$ acte et de la $1^{\text {re }}$ scène du $\mathrm{III}^{\mathrm{e}}$ acte, et le désir décuplé s'emparant des différents personnages, sont autant de traductions différentes de l'agentivité de l'île. Il faut donc tenter de mettre au jour les mécanismes musicaux déployés par Schreker pour placer l'auditeur - intradiégétique ou extradiégétique - dans l'état de stase érotique initié par le tableau vivant inaugurant l'acte. Le Klang constitue l'un de ces outils essentiels; le terme renvoie plus précisément à un complexe timbre-son caractérisé par sa ductilité, que Christopher Hailey, dans sa monographie consacrée à Schreker, définit de la sorte :

Klang est l'un de ces mots si riches en connotation qu'il n'a pas d'unique équivalent en anglais. "Son", "bruit", "note", "ton", "couleur", "timbre", "tintement" et "carillon" font chacun partie de son sens; il est rare que seul un de ces sens soit convoqué. Dans la musique de Schreker, le terme fait généralement référence à une combinaison d'orchestration (doublages subtils et effets instrumentaux) et à une ambiguité harmonique (sonorités avec deux racines fonctionnelles, tons non harmoniques ajoutés, basse indéfinie) ${ }^{57}$.

Adorno semble en avoir le premier saisi toutes les dimensions, lorsqu'il évoque ce phénomène qui

fait naître l'image d'un son qui en quelque sorte ne vient de nulle part et ne va nulle part. Il est là tout d'un coup, comme si on faisait vibrer des cordes, suspendu dans les airs tel un mirage auditif, diapré, transparent, dénaturé. Il reste insaisissable et 
s'évanouit. Véritable fantasmagorie, il cherche à arracher la musique au temps, à la traduire dans l'espace ${ }^{58}$.

Ces fantasmagories sonores du Klang interviennent de manière particulièrement frappante à deux reprises dans l'opéra : le prélude du I $^{\mathrm{er}}$ acte, et celui du $\mathrm{III}^{\mathrm{e}}$ acte. Dès les premières mesures de l'opéra, la singularité de Schreker s'affirme, et déploie une texture orchestrale ondoyante érigeant l'ambivalence - tonale, harmonique, timbrale en principe. Schreker s'emploie en effet à de savants jeux de mélanges de timbres, au moyen d'ostinatos entre différents instruments; dès les premières mesures, les instruments à cordes frappées (claviers, célesta), frottées (violons), et pincées (harpes) ${ }^{59}$ créent un son miroitant qui subsume les différents timbres des instruments le composant: le Klang. Cette esthétique de l'ambivalence chère à Schreker s'appuie également sur la complémentarité rythmique ternaire-binaire au sein des pupitres (entre les deux parties du clavier, les deux parties de harpe, de célesta, ou les deux pupitres de violons) ; elle contribue ainsi à renforcer cet effet de brouillage. Enfin, l'ambivalence harmonique constitue peut-être l'effet le plus prégnant de cette ambivalence : les mesures 1 à 3 sont en effet l'occasion d'accords de ré majeur (clavier, voix 1 ; célesta, voix 2 ; violons 2 voix 1 ) et de si $b$ mineur (violons 2 , voix 2 ; célesta, voix 2). L'effet se poursuit aux mesures suivantes, avec une ambivalence de do majeur et sol $b$ majeur pour la mesure 4, et une ambivalence mi majeur et sol b majeur ou fa\# majeur pour les mesures 5 à 8 . De ce tapis brumeux émerge un thème, lyrique et tragique, énoncé aux pupitres de violoncelles, d'altos, et de clarinette basse ; il s'agit du leitmotiv d'Alviano (cf. ill. 1) ${ }^{60}$.

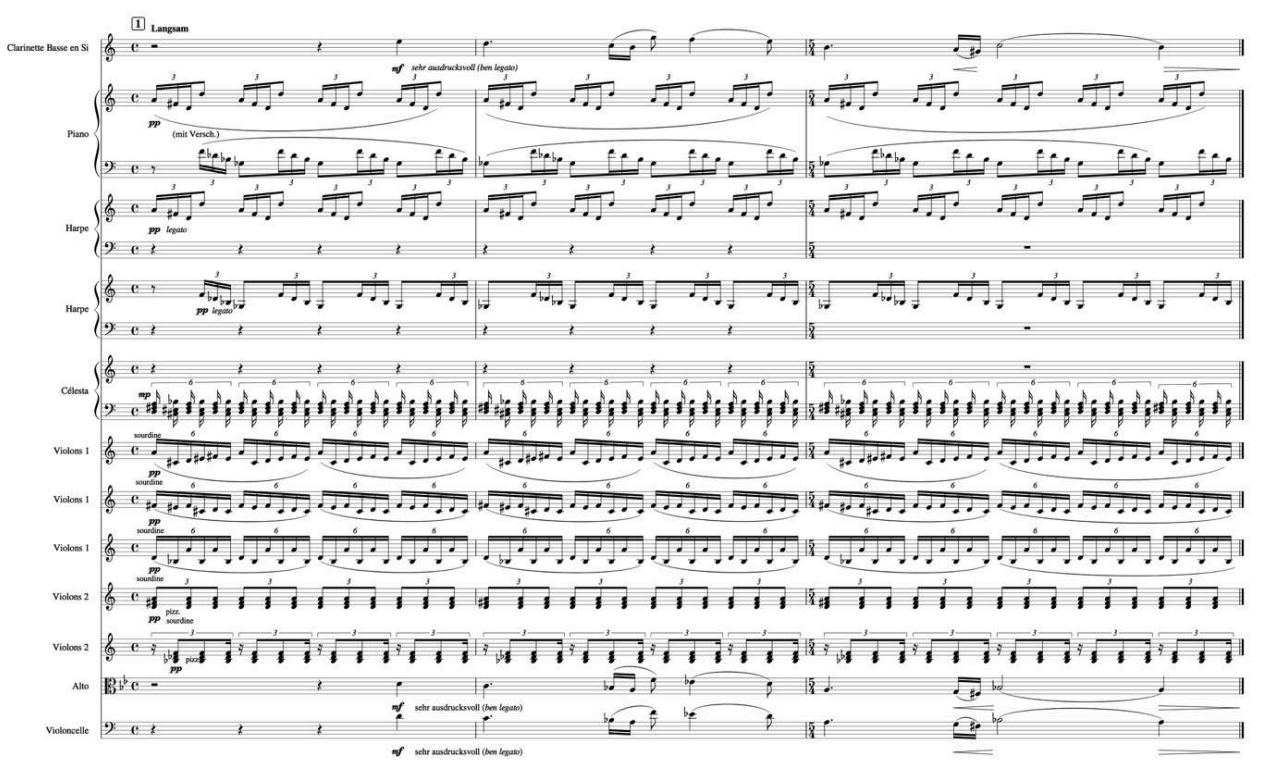

III. 1 : Die Gezeichneten, Acte I, mesures 1-3 sonore recourt aux mêmes procédés d'ostinatos fondés sur l'oscillation de couleurs harmoniques en apparence inconciliables. Les mesures 118 et 119 laissent en effet entendre à l'état embryonnaire une harmonie de septième mineure sur réb (ostinato 
des parties de Célesta ( $2^{\mathrm{e}}$ voix), Violons I ( ${ }^{\mathrm{e}}$ et $4^{\mathrm{e}}$ voix) et Violons II ( ${ }^{\mathrm{e}}$ voix), entièrement réalisée, par enharmonie, à la mesure 120 grâce au solo de la Harpe $\left(1^{\text {re }}\right.$ voix). L'intrication des différentes lignes instrumentales brouille néanmoins cette lecture, pour proposer une nébuleuse dont l'auditeur semble ne pouvoir se dépêtrer : les notes $f a-s o l-l a$, principalement jouées par le Piano ( $1^{\text {re }}$ voix), le Célesta ( $1^{\text {re }}$ voix) et les Violons $\mathrm{I}$ ( $1^{\text {re }}$ et $3^{\mathrm{e}}$ voix), ne peuvent en effet s'intégrer à cette harmonie. Là encore, ces textures s'appuient sur l'irrégularité des différentes divisions métriques précédemment évoquée (cf. ill. 2) comme pour « donner corps, par l'ivresse qu'elles procurent, à la folle chimère de tiédeurs lustrales, d'un chaos venu de l'âge des hétaïres - d'une musique qui n'aurait pas de contours précis, dans quelque dimension que ce soit ${ }^{61}$.

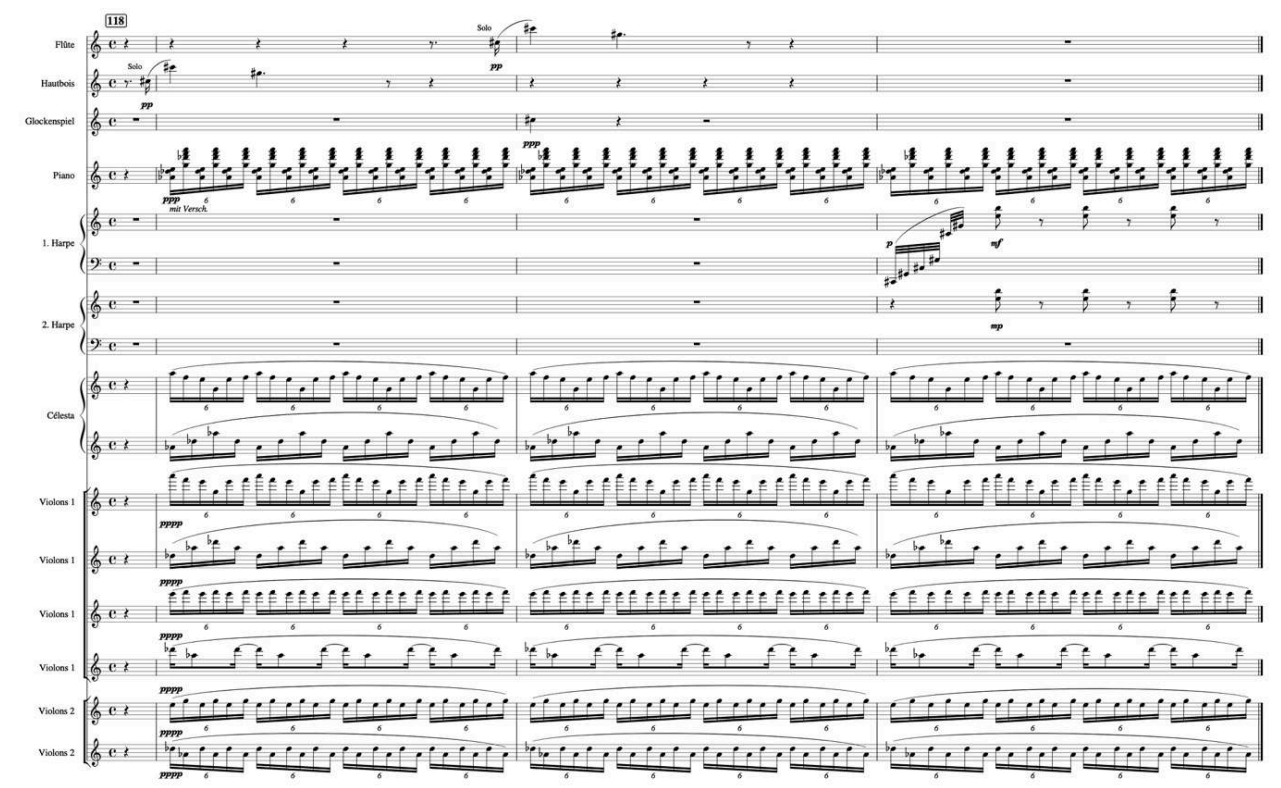

\section{2 : Die Gezeichneten, Acte III, mesures 118-12062} extradiégétiques, le son schrékerien repose également sur un procédé de spatialisation lui-même étroitement lié à la fascination érotique. Lier spatialisation et érotisme peut sembler surprenant au premier abord, tant ce procédé est surtout analysé pour la richesse acoustique dont il est souvent le signe, les esthétiques révolutionnaires qu'il illustre, ou les fonctions narratives qu'il supporte. Il est pourtant essentiel dans Les Stigmatisés: tout concourt à perdre l'auditeur, à le diluer dans un espace-temps insondable. Adrian Daub, dans son essai Tristan's Shadow parle ainsi d'" acoustique érotique $»^{63}$ pour désigner toute texture orchestrale où la musique devient une "fantasmagorie acoustique [...], spatialisée », qui « s'immobilise dans un entrelacement du près et du lointain aussi trompeur que la consolante fée Morgane $»^{64}$.

Parce qu'elles permettent la stase, la spatialisation et l'ambivalence, les «élixirs schrékeriens $»^{65}$ suscitent chez l'auditeur confusion et brouillage des objets distincts, dissolution de son être, puis identification avec la source érotique. L'univers clos et autoréférentiel créé par ces "élixirs» statiques participe de l'énergétique érotique déployée par l'île sur tous les plans. Tout fonctionne pour que le spectateur intra ou 
extradiégétique, fasciné, soit pris au piège de ce qui constitue pour Adrian Daub un véritable Zauberkreis, un cercle magique sans échappatoire ${ }^{66}$. Véritable "nébuleuse " qui «aspire toutes les figures", cette musique, rappelle Martin Kaltenecker, "n'en reste pas à la simple idée d'une telle utopie: elle invite l'auditeur à en gagner directement le rivage ${ }^{67}$. Prisonnier d'images et de sons contre lesquels il ne peut rien dans « une sorte de surréalisme inconscient [où] la distance esthétique est abolie " ${ }^{68}$, le spectateur régresse dans le néant; il connaît alors la transe ${ }^{69}$ caractéristique de l'érotisme sacré ou mystique que Bataille théorise dans son essai sur l'érotisme :

Toujours, c'est d'un détachement par rapport au maintien de la vie qu'il s'agit, de

l'indifférence à tout ce qui tend à l'assurer, de l'angoisse éprouvée dans ces conditions jusqu'à l'instant où les puissances de l'être chavirent, enfin de l'ouverture à ce mouvement immédiat de la vie qui est d'habitude comprimé, qui se libère soudain dans le débordement d'une joie infinie ${ }^{70}$.

31 Nul n'en est dupe : la portée des images suscitées par l'Élysée et celle des troublantes sonorités de l'orchestre excèdent le niveau intradiégétique de l'œuvre. L'action de l'île sur les personnages du drame est ainsi celle que le compositeur souhaite susciter sur son propre public; elle constitue également une métaphore de la pratique compositionnelle de Schreker, invariablement présentée comme une réponse à un stimulus extérieur ${ }^{71}$. Le récit de la genèse d'Irrelohe est dans cette perspective particulièrement éclairant: le compositeur relate, dans un article intitulé "Über die Entstehung meiner Opernbücher" ("À propos de la genèse de mes livrets d'opéra »), comment le surgissement d'un nom de gare, Irrenlohe, se transforme immédiatement en inspiration musicale :

Il devient alors clair à mes yeux que ce nom, dont je ne souhaitais pas connaître l'étymologie sans doute prosaïque, portait en lui les germes d'un drame. Et il en alla ainsi. L'opéra sur lequel je travaille présentement porte ce nom ; son livret a été écrit en trois jours. Irrelohe - flammes de la folie ${ }^{72}$.

\section{Conclusion}

Nous espérons, au terme de cet article, avoir réussi à faire entendre une lecture des Stigmatisés qui, plutôt que de prendre l'œuvre comme prétexte pour un métadiscours, analyse les mécanismes inédits et fascinants qu'elle déploie. En nous concentrant sur le livret et la partition de l'opéra, nous avons exclu de facto une dimension pourtant essentielle pour appréhender l'œuvre dans sa complexité: les différentes mises en scène qu'elle a suscitées. Il n'existe pas à notre connaissance de travaux portant spécifiquement sur les mises en scène contemporaines de la création des Stigmatisés; les articuler aux analyses consacrées au livret ou à la partition permettrait assurément d'enrichir notre compréhension de cette œuvre. Plus proches de nous, trois productions retiennent notre attention : celle proposée par le Festival de Salzburg en $2005^{73}$, celle de l'opéra de Lyon $^{74}$ en 2015 dans le cadre du festival «Les jardins enchantés $»^{75}$, et celle de l'Opéra de Zürich ${ }^{76}$ en 2018. Mettant en exergue la dimension fantastique du livret, la mise en scène de l'opéra de Lyon propose un espace sombre, traversé par les jeunes nobles accoutrés de tenues associées aux pratiques BDSM, et par des créatures mi-humaines, mi-animales. Retenant principalement de l'île un «jeu sauvage en référence à Bacchus ${ }^{77}$, elle met l'accent sur les crimes commis par Tamare et ses comparses, ce que soulignait, dès le début de l'opéra, la projection d'appels à témoin associés à chaque disparition de jeune femme. Quelques années plus tôt, la mise 
en scène de Salzburg témoignait d'un parti-pris très différent: dépassant les agissements des nobles, l'Élysée est avant tout un espace profondément ambivalent et inquiétant, peuplé de longues créatures encapées et masquées, ou uniquement vêtues de strings. Une sculpture antique de femme alanguie recouvre l'entièreté de la scène bordée de coursives étagées, faiblement éclairées; elle constitue l'unique décor de l'acte. La production de l'Opéra de Zürich, enfin, se concentre quant à elle sur la représentation omniprésente du désir sexuel. Figuré par les gestes extrêmement suggestifs des personnages, il est constamment rappelé par le pétrissage sensuel de la glaise ou les jeux érotiques que ce matériau suscite ; reliant les différents actes entre eux - de peintre, Carlotta devient sculptrice -, la métaphore de l'artiste rendu fou de désir pour sa création fait d'Alviano un nouveau Pygmalion.

\section{BIBLIOGRAPHIE}

ADORNO Theodor Wiesengrund, Quasi una fantasia : Écrits musicaux II, LELEU Jean-Louis (trad.), Paris, Gallimard, 1982 [1963].

ADORNO Theodor Wiesengrund, Essai sur Wagner, HILDENBRAND Hans et LINDENBERG Alex (trad.), Paris, Gallimard, 1966.

BATAILlÉ Christophe, « Pour un animisme poétique : “Tête de faune” ", Parade sauvage, nº 17/18, 2001, p. 106-119.

BATAILLE Georges, L'érotisme, Les Éditions de Minuit, 2011.

BREDEKAMP Horst, Théorie de l'acte d'image, Paris, éditions La Découverte, 2015.

BUJURA Karsten, "Klang - Farbe - Geschlecht - Sexualität : Diskursive Metaphorik nationaler Identität / Alterität in der Rezeptionsgeschichte der musikalischen Moderne am Beispiel des Komponisten Franz Schreker" Humboldt-Universität zu Berlin, Berlin, 2015.

Cosinschi Micheline, « Singularités » géotopiques de l'Imago Mundi - III, https://

www.researchgate.net/publication/

279193913_SINGULARITES_GEOTOPIQUES_DE_L'IMAGO_MUNDI_-IIIi.

DAHLHAUS Carl, "Schreker and modernism: on the dramaturgy of Der ferne Klang", Schoenberg and the New Music: Essays by Carl Dahlhaus, PUFFETT Derrick et CLAYTON Alfred (trad.), Cambridge, Cambridge University Press, 1987 [1978].

DAUB Adrian, Tristan's Shadow: Sexuality and the Total Work of Art after Wagner, Chicago, University of Chicago Press, 2013

DAUB Adrian, “Adorno's Schreker: Charting the Self-Dissolution of the Distant Sound”, Cambridge Opera Journal, vol. 18, n 3, 2006, p. 247-271.

DENUT Eric, « De Schœnberg à Deutsch : arguments pour un "sécessionnisme" musical », http:// www.schreker.org/neu/bibliographie/lesen/denut1.pdf, consulté le 2 septembre 2020.

DREYFUS Laurence, Wagner and the Erotic Impulse, Cambridge, Harvard University Press, 2012. 
FLAMION Aurore, « Rester en retrait de l'espace médiatique : le cas Schreker », Revue musicale OICRM, vol. 7, n 1, 2020, p. 142-159.

FLAMION Aurore, «Les Stigmatisés de Franz Schreker : entre décadence et dégénérescence, généalogie d'un récit idéologique », Mémoire de master de musicologie, Université Lyon 2, 2019. FRANKLIN Peter, “Lost in Spaces: Recovering Schreker's Spectacular Voices”, The Opera Quarterly, vol. $29, \mathrm{n}^{\circ} 1,2013$, p. 19-30.

FRANKLIN Peter, “'Wer weiss, Vater, ob das nicht Engel sind ?' Reflections on the Pre-Fascist Discourse of Degeneracy in Schreker's Die Gezeichneten", BACHT Nikolaus (dir.), Music, theatre and politics in Germany: 1848 to the Third Reich, Aldershot; Burlington, Ashgate, 2006.

GELL Alfred, L'art et ses agents - une théorie anthropologique, Dijon, Les presses du réel, 2009.

HAILEY Christopher, "Franz Schreker and the Pluralities of Modernism", Tempo, n 219, 2002, http://www.jstor.org/stable/946695.

HAILEY Christopher, Franz Schreker, 1878-1934: A Cultural Biography, Cambridge, 1993.

HALIMI Carole, « La "sensation magique" du tableau vivant ou la recherche d'une image active ", KESSLER Frank, LARRUE Jean-Marc et PISANO Giusy (dir.), Machines. Magie. Médias., Villeneuve d'Ascq, Presses universitaires du Septentrion, 2019, p. 383-398.

HATTEN Robert S., A theory of virtual agency for Western art music, Bloomington, Indiana, Indiana University Press, 2018.

HERBERT Marcuse, Eros et civilisation, NÉNY Jean-Guy et FRAENKEL Boris (trad.), Les Éditions de Minuit., Paris, 1963.

KALTENECKER Martin, L'Oreille divisée : les discours sur l'écoute musicale aux XVIII et XIX ${ }^{e}$ siècles, Paris, Musica Falsa, 2010.

LE BRUN Annie, Un espace inobjectif : entre les mots et les images, Paris, Gallimard, 2019.

LEDUC Alain, « Les hyperboles du narcissisme dans le livret des Stigmatisés de Franz Schreker », Germanica, $\mathrm{n}^{\circ} 41,2007$, p. 45-61.

LEDUC Alain, « Le lien entre musique et inconscient dans Das Spielwerk und die Prinzessin de Franz Schreker », Germanica, nº 36, 2005, p. 45-65.

LEE Sherry D., ““...deinen Wuchs wie Musik': Portraits, Identities, and the Dynamics of Seeing in Berg's Operatic Sphere", HAILEY Christopher (dir.), Alban Berg and His World, Princeton, Princeton University Press, 2010, p. 163-194.

LEE Sherry D., "A Minstrel in a World without Minstrels: Adorno and the Case of Schreker", Journal of the American Musicological Society, vol. 58, n 3, 2005, p. 639-696.

Les Stigmatisés - l'École du spectateur, Lyon, 2015.

PERROUX Alain, Franz Schreker ou À la recherche du son lointain, Drize, Papillon, 2001.

PICARD Timothée, L'art total, grandeur et misère d'une utopie (autour de Wagner), Rennes, Presses universitaires de Rennes, 2006.

RYKNER Arnaud, Corps obscènes : Pantomime, tableau vivant et autres images pas sages : suivi de Note sur le dispositif, Mulhouse, Orizons, 2014.

SCHREKER Franz, Die Gezeichneten, München, Höflich, 2011, vol. 2. 
SCHREKER Franz, “Über die Entstehung meiner Opernbücher”, Musikblätter des Anbruch, vol. 2, $\mathrm{n}^{\circ} 16,1920$ p. 547-549.

SCHREKER Franz, “Meine Musikdramatische Idee”, Musikblätter des Anbruch, vol. I, nº 1, 1919, p. 6-7. SIMON Agathe, « Georges Bataille, le plaisir et l'impossible », Revue d'histoire littéraire de la France, vol. 103, nº 1, 2003, p. 181-186.

SPENLEHAUER Jean (dir. et trad.), Les Stigmatisés : Franz Schreker, Lyon, Opéra national de Lyon, 2015.

STEINKE Tim, "Franz Schreker: Die Gezeichneten", Oper nach Wagner: Formale Strategien im europäischen Musiktheater des frühen 20. Jahrhunderts, Kassel, Bärenreiter, 2011, p. 205-293.

TODOROV Tzvetan, Poétique de la prose, Paris, Seuil, 1980.

vouilloux Bernard, Le tableau vivant : Phryné, l'orateur et le peintre, Paris, Flammarion, 2015.

\section{NOTES}

1. ADORNo Theodor Wiesengrund, Quasi una fantasia: Écrits musicaux II, LELEU Jean-Louis (trad.), Paris, Gallimard, 1982 [1963], p. 148.

2. Sur l'analyse de l'œuvre de Schreker par Adorno, voir LEE Sherry D., "A Minstrel in a World without Minstrels: Adorno and the Case of Schreker", Journal of the American Musicological Society, vol. 58, n $\mathrm{n}^{\circ}$ 3, 2005, p. 639-696 ; DAUB Adrian, “Adorno's Schreker: Charting the Self-Dissolution of the Distant Sound", Cambridge Opera Journal, vol. 18, n 3, 2006, p. 247-271.

3. Voir DahlHaus Carl, "Schreker and modernism: on the dramaturgy of Der ferne Klang", Schoenberg and the New Music: Essays by Carl Dahlhaus, PUFFETT Derrick et CLAYTON Alfred (trad.), Cambridge, Cambridge University Press, 1987 [1978] ; HAILEY Christopher, Franz Schreker, 1878-1934: a cultural biography, Cambridge, 1993 ; BUjURA Karsten, "Klang - Farbe - Geschlecht - Sexualität : Diskursive Metaphorik nationaler Identität / Alterität in der Rezeptionsgeschichte der musikalischen Moderne am Beispiel des Komponisten Franz Schreker", Humboldt-Universität zu Berlin, Berlin, 2015. Nous avons tenté, dans le cadre d'un mémoire de master, de reconstituer les différents récits (genré, antisémite, politique, historique) pouvant expliquer la désaffection pour Schreker des dernières décennies: FLAMION Aurore, "Les Stigmatisés de Franz Schreker: entre décadence et dégénérescence, généalogie d'un récit idéologique », Mémoire de master de musicologie, Université Lyon 2, 2019.

4. L'article d'Éric Denut consacré au sécessionnisme musical de Schreker donne une bonne idée des discours portant sur la profusion stylistique de Schreker: DENUT Éric, «De Schœnberg à Deutsch: arguments pour un "sécessionnisme" musical », http://www.schreker.org/neu/ bibliographie/lesen/denut1.pdf, consulté le 2 septembre 2020.

5. Elle inspire également à Schreker un ballet-pantomime éponyme, en 1908. Le conte d'Oscar Wilde constitue pour Christopher Hailey, auteur d'une monographie de référence consacrée à Schreker, la source principale d'inspiration de ce dernier pour cet opéra. Albert Gier, dans son article « Franz Schreker, compositeur-librettiste », rapproche également le livret des Contes cruels de Villiers de L'Isle-Adam, parus en 1904 dans une édition allemande et offrant un grand nombre de similitudes avec Les Stigmatisés. Est ainsi racontée l'histoire du cinquième duc de Portland (1800-1879), personnage historique devenant sous la plume de l'écrivain un «viveur, jeune et insouciant qui, au cours d'un voyage en Orient, serre la main d'un mendiant lépreux et se voit atteint de la même maladie. Revenu en Angleterre [...] il fait procéder à un splendide aménagement des caves du château, qu'il orne de miroirs vénitiens [etc.] avant d'inviter la “jeunesse dorée” à s'y livrer à des fêtes libertines auxquelles, cela va de soi, il s'abstient de 
prendre part »; Albert Gier, « Franz Schreker, compositeur-librettiste », dans Bernard Banoun et Jean-François Candoni (dir.), Le monde germanique et l'opéra. Le Livret en question, Paris: Klincksieck, 2005, p. 277-293, p. 290.

6. Le lied "Der Zwerg" de Franz Schubert, composé dans les années 1820 sur un texte de Matthäus von Collin, met en scène un nain qui, tombé amoureux d'une reine, la tue lorsqu'il comprend qu'elle l'a délaissé pour un autre ; le lied se clôt sur son propre suicide.

7. Tim Steinke voit en Alviano un nouveau Rigoletto : tous deux sont infirmes, nobles de cœur et responsables de leur propre malheur. Tamare, le rival d'Alviano, hériterait quant à lui du Duc : orgueilleux, puissants, ils sont incapables d'amour sincère; STEINKE Tim, "Franz Schreker: Die Gezeichneten", Oper nach Wagner: Formale Strategien im europäischen Musiktheater des frühen 20. Jahrhunderts, Kassel, Bärenreiter, 2011, p. 205-293, p. 260.

8. Acte III, Scène 1; SPENLEHAUER Jean (dir. et trad.), Les Stigmatisés : Franz Schreker, Lyon, Opéra national de Lyon, 2015, p. 117.

9. "His orchestration, harmonic language, motivic design, and structural organization are uniquely his own and owe little to Brahms or Wagner, or to that synthesis of the two that we hear in the early works of Zemlinsky and Schoenberg. One is tempted to posit the influence of French Impressionism but aside from Charpentier's Louise the composer knew little contemporary French music at this time. Schreker seems to emerge from nowhere with a wholly idiosyncratic musical language, whose originality lies less in a fusion of impulses than in a daring montage of often clearly identifiable sources" ; HAILEY Christopher, "Franz Schreker and the Pluralities of Modernism", Tempo, n 219, 2002, http://www.jstor.org/stable/ 946695 , p. 4.

10. Sur le rapport de Schreker avec la presse voir FLAMION Aurore, « Rester en retrait de l'espace médiatique : le cas Schreker », Revue musicale OICRM, vol. 7, n 1, 2020, p. 142-159.

11. 11 ADORNo, Quasi Una Fantasia, p. 153.

11. Alfred Gell considère ainsi qu'un « objet est animé parce que nous lui attribuons non pas une vie biologique mais une subjectivité/intentionnalité »; GELL Alfred, L'art et ses agents - une théorie anthropologique, Dijon, Les Presses du réel, 2009 [1998], p. 150.

12. HATTEN Robert S., A theory of virtual agency for Western art music, Bloomington (Indiana), Indiana University Press, 2018.

13. "For my purpose, a virtual agent in music is not an actual agent, but its efficiency lies in its capacity to simulate the actions, emotions, and reactions of a human agent." Ibid., p. 1.

14. "It's important to note that such interpretations are not just subjective projections but can be intersubjective (shared) inferences that are stylistically and strategically warranted by specific features of a musical work.", Ibid., p. 2.

15. "I propose such virtual agency even in the absence of verbal evidence of its envisioning by composers, or manifestation by performers" Ibid., p. 7.

16. BATAILlE Georges, L'érotisme, Les Éditions de Minuit, 2011, p. 35.

17. Agathe Simon rappelle ainsi que la dialectique érotique du plaisir et de "l'imminence perpétuelle " - mais impossible - de sa réalisation entraîne, chez Bataille, une focalisation à l'extrême de la conscience sur cette possibilité de rupture: sIMON Agathe, «Georges Bataille, le plaisir et l'impossible », Revue d'histoire littéraire de la France, vol. 103, n 1 1, 2003, p. 181-186, p. 186. 18. Sur la musique des Stigmatisés voir STEINKE, "Franz Schreker: Die Gezeichneten". Sur son contenu dramaturgique, voir DAUB Adrian, Tristan's Shadow: Sexuality and the Total Work of Art after Wagner, Chicago, University of Chicago Press, 2013 ; DAUB, “Adorno's Schreker” ; FRANKLIN Peter, “Wer weiss, Vater, ob das nicht Engel sind?' Reflections on the Pre-Fascist Discourse of Degeneracy in Schreker's Die Gezeichneten", BACHT Nikolaus (dir.), Music, theatre and politics in Germany: 1848 to the Third Reich, Aldershot ; Burlington, Ashgate, 2006 ; FRANKLIN Peter, "Lost in Spaces: Recovering Schreker's Spectacular Voices", The Opera Quarterly, vol. 29, $\mathrm{n}^{\circ} 1,2013$, p. 19-30 ; LEE, "A Minstrel in a World without Minstrels"; LEE Sherry D., “....deinen Wuchs wie 
Musik': Portraits, Identities, and the Dynamics of Seeing in Berg's Operatic Sphere", HAILEY Christopher (dir.), Alban Berg and His World, Princeton, Princeton University Press, 2010, p. 163-194.

19. Herbert Marcuse, dans son exégèse de Freud, rappelle ainsi que «la régression impliquée dans un tel développement de la libido se manifest[e] d'abord par une activation de toutes les zones érotiques et donc par la renaissance de la sexualité polymorphe prégénitale et par le déclin de la suprématie génitale. Tout le corps devien[t] un objet de cathexis, une chose pour jouir, un instrument de plaisir.»; HERBERT Marcuse, Eros et civilisation, NÉNY Jean-Guy et FRAENKEL Boris (trad.), Les Éditions de Minuit, Paris, 1963, p. 191.

20. Réduit à quelques figures anonymes brièvement croisées dans l'économie de l'opéra ( «le Premier Bourgeois ", « le Deuxième Bourgeois ", « un Petit Garçon », « le Père ", « la Mère ", « le Troisième Bourgeois »...) le peuple apparaît au début du $\mathrm{III}^{\mathrm{e}}$ acte quelque peu naïf, entièrement réduit aux impressions que l'île produit sur lui.

21. Acte III, Scène 1 ; SPENLEHAUER (dir. et trad.), Les Stigmatisés : Franz Schreker, p. 119.

22. CARLOTTA : Als hätt' ich Wein getrunken, / Schwer und vermischt mit behexenden Kräutern, / Oder jenen seltsamen Trank aus Herzblut / Einer liebtollen Jungfrau gebraut! / Es ist der Schönheit furchtbarer Zauber, / Gebunden sonst, halb verborgen den Sinnen, / Und nun entfesselt, preisgegeben dem Tage/ Und lüsternen Blicken ; Acte III, Scène 8 ; Ibid, p. 139.

23. Carlotta, au cours de la scène de l'atelier évoque la situation d'une amie peintre, souffrant d'un mystérieux trouble au cœur : «Elle avait sans doute une angoisse secrète, / Que dans sa vie survienne / Un jour un événement capital; / Quelque troublante merveille qui la ferait succomber ", Acte II, Scène 2 ; Ibid, p. 99. Il s'agit en réalité de sa propre histoire : Carlotta meurt après s'être donnée à Tamare sur l'Élysée (Acte III, Scène 20).

24. LEE, ““...deinen Wuchs wie Musik': Portraits, Identities, and the Dynamics of Seeing in Berg's Operatic Sphere", p. 180.

25. "Yet by the end of the opera she has become unmistakably a Weiningerian woman, whose complete surrender to sexuality overrides not only rationality and morality but lofty intellectual or creative ideals." ; Ibid, p. 180.

26. L'indépendance d'esprit de Carlotta était jusque-là symbolisée par son activité de peintre ; son père, le Podestat, la décrit en ces termes à Alviano, Acte I, Scène $4:$ « Je crains, seigneur, que vous la trouviez d'un esprit trop libre ; / Elle fait peu de cas, à mon grand regret, / Des normes de la société. »; SPENLEHAUER (dir. et trad.), Les Stigmatisés : Franz Schreker, p. 49.

27. Alviano parcourt tout le $\mathrm{III}^{\mathrm{e}}$ acte à la recherche de Carlotta, poursuivi par des hallucinations auditives : « Depuis hier, j'entends des voix partout / Des chants dans les airs, des murmures dans les buissons / Il y a peu le rire argentin de Carlotta / Mais c'était sans doute une illusion » (Acte III, Scène 5 ; Ibid, p. 129.) ; «comme traqué: J'entends sa voix! / elle me parvient des sommets, des ravins, multipliée par mille! Chant des airs, chansons moqueuses du fond de l'enfer! Son image danse devant moi, épie dans les buissons et les haies » (Acte III, Scène 16 ; Ibid, p. 165).

28. LEDUC Alain, « Les hyperboles du narcissisme dans le livret des Stigmatisés de Franz Schreker », Germanica, $\mathrm{n}^{\circ} 41,2007$, p. 45-61, \$26.

29. Ibid, §20.

30. RYKNER Arnaud, Corps obscènes : Pantomime, tableau vivant et autres images pas sages : suivi de Note sur le dispositif, Mulhouse, Orizons, 2014, p. 11.

31. vouilloux Bernard, Le tableau vivant: Phryné, l'orateur et le peintre, Paris, Flammarion, 2015, p. 55.

La proximité entre cette hypotypose et le tableau vivant est également rapportée par Arnaud Rykner, lorsqu'il rappelle que «silencieux et immobile, le tableau vivant, dans son utilisation la plus fréquente, laisse toutefois une place importante au texte, qu'il s'agisse de l'“hypotexte" qui 
lui donne souvent naissance (comme illustration visuelle d'un texte préexistant) ou qu'il s'agisse plus simplement des commentaires qui l'accompagnent. »; RYKNER, Corps obscènes, p. 168.

32. BREDEKAMP Horst, Théorie de l'acte d'image, Paris, La Découverte, 2015 [2007], p. 17.

33. HALIMI Carole, "La "sensation magique" du tableau vivant ou la recherche d'une image active », KESSLER Frank, LARRUE Jean-Marc et PISANO Giusy (dir.), Machines. Magie. Médias., Villeneuve d'Ascq, Presses universitaires du Septentrion, 2019, p. 383-398, p. 384.

34. L'échange entre le Podestat et Alviano, Acte III, Scène 5, est à cet égard éloquent :

Le PODESTAT (un peu évasif) : Je suis troublé, et ébloui. / Ce que vous venez de me montrer, c'est inconcevable / Que le cerveau d'un seul homme / Ait pu penser cet empire du merveilleux !

ALVIANO : Beaucoup m'ont aidé. / Les artistes ont agi, je n'ai donné que le désir ! ; SPENLEHAUER (dir. et trad.), Les Stigmatisés : Franz Schreker, p. 129.

35. HALIMI, «La "sensation magique" du tableau vivant ou la recherche d'une image active ", p. 391.

36. Le Troisième Bourgeois déclare ainsi gravement, Acte III, Scène 1 : «Voyez-vous, Madame, vous ne comprenez pas ça : / C'est de «l'art»! / Sous feu le doge Francesco Sforza, / Paix à son âme, c'était un seigneur sévère, / J'ai collaboré à un tableau dans ce genre ». Il ajoute quelques mesures plus loin : «Quand je suis arrivé et que j'ai vu tout ça, / J'ai repensé à cette peinture / Il y en a beaucoup au palais, mais ici, vraiment / C'est beaucoup plus beau »; SPENLEHAUER (dir. et trad.), Les Stigmatisés : Franz Schreker, p. 119.

37. Les Génois insistent dès leur arrivée sur l'île sur leurs impressions sensuelles. Au Troisième Bourgeois qui déclare trouver l'Élysée beaucoup plus belle qu'un tableau, le peuple répond:

LA MÈRE : Parce que tout vit.

LE DEUXIÈME BOURGEOIS : Et embaume.

LE PREMIER BOURGEOIS : Et toute cette lumière! Ibid, p. 121.

38. Nous n'avons pas connaissance d'écrits faisant état d'influences picturales chez Schreker; irriguant ses principaux opéras, elles pourraient assurément faire l'objet d'un travail de recherche.

39. LEDUC, « Les hyperboles du narcissisme dans le livret des Stigmatisés de Franz Schreker », \$25.

40. BATAILLÉ Christophe, "Pour un animisme poétique: "Tête de faune"», Parade sauvage, $\mathrm{n}^{\circ}$ 17/18, 2001, p. 106-119, p. 106.

41. Pour Bataille, « de l'orgie procède un aspect archaïque de l'érotisme. L'érotisme orgiaque est en son essence excès dangereux. Sa contagion explosive menace indistinctement toutes les possibilités de la vie »; BATAILLE, L'érotisme, p. 121.

42. BATAILLÉ, « Pour un animisme poétique », p. 116.

43. La didascalie indique à cet endroit: "Blasender Faun auf der Szene” (« Un faune musicien sur la scène »).

44. Dans la mythologie grecque, Éros condamne Pan, le dieu des bergers des pâturages et des bois, caractérisé par sa laideur et son appétit sexuel insatiable, à voir ses avances refusées par la nymphe Syrinx. Celle-ci préfère être changée en roseau plutôt que de céder à Pan; il se console en fabriquant une flûte à partir de roseaux.

45. Le musicologue écrit ainsi : «Que toute l'activité des faunes, des naiades et des nymphes se déroule explicitement à un niveau purement sexuel, c'est ce que souligne Schreker en rendant visible le faune soufflant dans sa syrinx. " ("Dass sich dabei das gesamte Treiben der Faune, Najaden und Nymphen explizit auf rein sexueller Ebene abspielt, macht Schreker durch den Hinweis auf die von einem Faun geblasene Syrinx sichtbar") ; T. STEINKE, "Franz Schreker: Die Gezeichneten", op. cit., p. 275.

46. Tzvetan Todorov l'avait rappelé, dans son court essai consacré à L'Odyssée : "Celui qui entend le chant des Sirènes ne pourra survivre: chanter signifie vivre si entendre égale mourir »; TODOROv Tzvetan, Poétique de la prose, Paris, Seuil, 1980 [1971], p. 26.

47. Les Stigmatisés - l'École du spectateur, Lyon, 2015. 
48. LE BRUN Annie, Un espace inobjectif: entre les mots et les images, Paris, Gallimard, 2019, p. 8-9.

49. "Glockenläuten aus der Stadt (Angelus) sehr entfernt, kaum vernehmbar" (" On entend au loin, venant de la ville, les cloches de l'angélus, à peine perceptibles »). L'importance de l'angélus dans cette scène n'est pas sans faire penser à l'opéra Tosca de Puccini, également parcouru ce signal ambivalent; la proximité stylistique que décèle Tim Steinke entre le leitmotiv de Tamare, dans les Stigmatisés, et le thème d'amour de Tosca pourrait étayer ce rapprochement volontaire de la part de Schreker. Voir Ibid., p. 218.

50. Voir FLAMION, « Rester en retrait de l'espace médiatique. Le cas Schreker ».

51. Schreker déclare en effet dans son article "Meine Musikdramatische Idee" : « la sonorité, quel mot méprisé, conspué - "que du son», "uniquement des sons »... Si les grincheux savaient quelles possibilités expressives, quelle magie sonore inouie sont renfermées dans une sonorité, un accord ! [...] Cette sonorité pure, sans aucun ajout de motifs, constitue, si on l'utilise avec précaution, l'un des moyens d'expression les plus essentiels en musique. » ("Klänge - welch arg missbrauchtes, vielgeschmähtes Wort! Nur ein Klang - nur Klänge! Wüssten die Nörgler, welche Ausdrucksmöglichkeiten, welch unerhörter Stimmungszauber ein Klang, ein Akkord in sich Bergen kann! [...] Der reine Klang, ohne jede motivische Beigabe ist, mit Vorsicht gebraut, eines der wesentlichsten musikdramatischen Ausdrucksmittel".); SCHREKER Franz, "Meine Musikdramatische Idee", Musikblätter des Anbruch, vol. I, $\mathrm{n}^{\circ}$ 1, 1919, p. 6-7. La préface du Carillon et la Princesse est également éloquente : «Appelez cela la "pulsion" que le Maître a placée dans tout ce qui vit, le miracle de la création dans son éternel renouvellement - ou bien appelez cela "sensualité", "concupiscence perverse" - c'est l'origine de tout le bien et de tout le mal, de la félicité et de la déception, du bonheur et de la misère, de la vie et de la mort : c'est le divin et le diabolique. »; cité dans LEDUC Alain, « Le lien entre musique et inconscient dans Das Spielwerk und die Prinzessin de Franz Schreker ", Germanica, n³ 36, 2005, p. 45-65, §33.

52. Tim Steinke considère ainsi que les représentations musicales de l'Élysée et de ses orgies sont des expressions récurrentes chez Schreker, que l'on retrouve dans le $\mathrm{II}^{\mathrm{e}}$ acte de Der Ferne Klang et dans le III acte de Der Schatzgräber ; STEINKE, "Franz Schreker: Die Gezeichneten".

53. Timothée Picard rappelle ainsi, dans son essai consacré à l'art total : " Dans certains opéras des compositeurs viennois Zemlinsky, Braunfels, et surtout Schreker, le lyrisme exacerbé, venant redoubler la peinture d'une érotique obsessionnelle, est la trace également d'une volonté déclarée de rivaliser avec l'Éros wagnérien, volonté qui peut parfois outrepasser son objet et se transformer en excès, saturation, et reflet grimaçant. »; PICARD Timothée, L'art total, grandeur et misère d'une utopie (autour de Wagner), Rennes, Presses universitaires de Rennes, 2006, p. 241.

54. KALTENECKER Martin, L'Oreille divisée: les discours sur l'écoute musicale aux XVIII et XIX ${ }^{\mathrm{e}}$ siècles, Paris, Musica Falsa, 2010, p. 391.

55. «Le Son lointain, Le Carillon, Les Stigmatisés ne sont pas trois livrets qui se suivent de manière arbitraire, l'auteur ne choisit pas au hasard tel ou tel contenu. Ce sont trois fantaisies sur le même sujet et ce sujet est le son. Il prend la forme symbolique d'un appel mystérieux, de la beauté, de l'élan vital dévastateur, il s'exprime dans la toute-puissance des sens, fascinante et enivrante, qui transforme les âmes et les recréée.»; Bekker, cité dans LEDUc, "Le lien entre musique et inconscient dans Das Spielwerk und die Prinzessin de Franz Schreker », §37.

56. Ibid. \$36. Schreker se situe ici dans la droite lignée de Wagner, dont Laurence Dreyfus rappelle dans son ouvrage Wagner and the Erotic Impulse qu'il «se distingue de ces prédécesseurs en matière d'opéra qui, malgré leur musique merveilleuse, observent le désir sexuel à une distance esthétique plus sûre. " ("Wagner distinguishes himself from his operatic predecessors who, for all their wonderful music, observe sexual desire from a safer aesthetic distance.") ; DREYFUS Laurence, Wagner and the Erotic Impulse, Cambridge (Ma.), Harvard University Press, 2012, p. 2.

57. "Klang is one of those words so rich in connotation that there is no single English equivalent. 'Sound', 'noise', 'note', 'tone', 'colour', 'timbre', 'ring(ing)', and 'peal(ing)' are each part of its meaning and there is 
seldom an instance where only one of these meanings is implied. In Schreker's music the term usually refers to a combination of orchestration (subtle doublings and instrumental effects) and harmonic ambiguity (sonorities with two functional roots, added non-harmonic tones, indefinite bass)"; HAILEY, Franz Schreker, p. 49.

58. ADORNO, Quasi Una Fantasia, p. 151.

59. On peut alors rappeler les propos d'Adorno à ce sujet : «Le mot "Klang”, en lui-même, évoque d'abord la harpe ", Ibid., p. 152.

60. Pour une lecture plus complète du prélude, nous renvoyons au conducteur disponible sur imslp : $\quad$ https://ks.imslp.net/files/imglnks/usimg/3/32/IMSLP35541-PMLP79751Schreker_Gezeichneten_Vorspiel.pdf

61. ADORNo, Quasi Una Fantasia, p. 153.

62. SCHREKER Franz, Die Gezeichneten, München, Höflich, 2011, vol. 2, p. 21.

63. DAUB, Tristan's Shadow, p. 96.

64. ADORNO Theodor Wiesengrund, Essai sur Wagner, HILDENBRAND Hans et LINDENBERG Alex (trad.), Paris, Gallimard, 1966, p. 115.

65. ADORNo, Quasi Una Fantasia, p. 153.

66. DAUB, “Adorno's Schreker”, p. 256. Le cercle convoque l'île et l'utopie ; or, l'île - de L'Odyssée, en passant par l'Utopie de Thomas More (1516), pour n'en citer que les exemples les plus emblématiques - est interprétée de manière récurrente comme étant le «seul véritable symbole originel ». En effet, pour Micheline Cosinschi, « dans son ensemble l'image de l'île, et donc du monde (y compris l'orbis terrarum), nous rappelle l'organe matriciel originaire. Ulysse est prisonnier des "grottes profondes" de Calypso (Homère: Chant I) »; cosinschI Micheline, "Singularités» géotopiques de l'Imago Mundi - III, https://www.researchgate.net/publication/ 279193913_SINGULARITES_GEOTOPIQUES_DE_L'IMAGO_MUNDI_-IIIi.

67. KALTENECKER, L'Oreille divisée. Les discours sur l'écoute musicale aux XVIII et XIX ${ }^{e}$ siècles, p. 155.

Adorno, dans une perspective très proche, avait déclaré dans sa conférence de 1959: "l'idéal sonore de Schreker est celui d'une musique qui jette des racines aériennes. Elle ne connaît ni origine ni histoire, et par-dessus tout refuse cette détermination de la musique qui est proprement le fait de la composition. »; ADORNo, Quasi Una Fantasia, p. 151.

68. ADORNo, Quasi Una Fantasia, p. 155.

69. Le reste du $\mathrm{III}^{\mathrm{e}}$ acte met en évidence les effets produits par ces mixtures orchestrales. Rapidement en effet, les Génois se mêlent aux fantasmagories de l'île, comme en témoigne le leitmotiv "Ah, welche Nacht", énoncé pour la première fois par Carlotta (Acte III, Scène 11, mesure 518), repris par certains des jeunes nobles se livrant à des orgies (mesure 533 , hors scène) et rapidement énoncé par le chœur : mesure 554, chanté appassionato par les basses, mesure 586 avec une écriture en imitation des différentes voix, pour atteindre mesure 600 un tutti extatique chanté "mit hochstem Ausdruck, größter fanatischer Leidenschaft". Ce leitmotiv surgit notamment de façon significative lors de l'abandon de Carlotta à Tamare, indiquant que l'acte charnel a eu lieu. 70. BATAILLE, L'érotisme, p. 253.

71. Nous renvoyons aux articles écrits par Schreker lui-même, qui donnent un aperçu du regard réflexif porté sur sa pratique compositionnelle: SCHREKER, "Meine Musikdramatische Idee »; SCHREKER Franz, "Über die Entstehung meiner Opernbücher », Musikblätter des Anbruch II/16, 10/1920 p. 547-549.

72. Le mot "Irre" signifie folie en allemand; "Lohe" désigne quant à lui les flammes. SCHREKER, "Über die Entstehung meiner Opernbücher"; PERROux Alain, Franz Schreker ou À la recherche du son lointain, Drize, Papillon, 2001, p. 80.

73. Mise en scène de Nikolaus Lehnhoff, direction musicale de Kent Nagano.

74. Mise en scène de David Bösch, direction musicale d'Alejo Pérez. 
75. Se tenant du 13 au 29 mars 2015, le festival avait également programmé Orphée et Eurydice de Gluck, et Le Jardin englouti de Michel Van der Aa.

76. Mise en scène de Barrie Kosky, direction musicale de Vladimir Jurowski et Giedrè Slekytė.

77. Propos de Faklo Herold, créateur des costumes de la production lyonnaise, dans une vidéo de médiation mise en ligne par l'opéra: https://www.youtube.com/watch? $\mathrm{v}=$ Ok0xUPPiyVc\&list=PLWoadw5rtauRPAh8NT5d35BebYrw7AuVK\&index=2

\section{RÉSUMÉS}

Créé en 1918, l’opéra Die Gezeichneten («Les Stigmatisés ») de Franz Schreker a suscité de nombreux discours fustigeant l'omniprésence d'une sexualité débridée et délétère, s'attachant indistinctement au sujet, à la musique ou au compositeur lui-même. L'œuvre met en scène la tragédie d'un jeune noble, laid, qui ayant cru en la rédemption par l'amour, sombre dans la folie après avoir cédé à la ville de Gênes une île appelée « Élysée » et dédiée aux plaisirs charnels.

L'ambition de cet article est de redonner une place au matériau opératique lui-même: paradoxalement réduite à l'anecdotique dans sa réception contemporaine, l'œuvre déploie pourtant des mécanismes puissants qui, illustrés par la mise en abyme de la venue des Génois sur l'île, agissent avec force sur les spectateurs. Il s'agit de mettre au jour en deux temps les moyens déployés par l'opéra pour imposer au spectateur intra-diégétique - et par extension, au public de l'œuvre - une stase qui se révèle être profondément érotique. La première partie, consacrée à l'esthétique du tableau vivant que convoque le $\mathrm{III}^{\mathrm{e}}$ acte, analyse les différentes modalités de la subjugation visuelle à laquelle est soumis le peuple génois. La deuxième partie poursuit cette analyse de la stase érotique sur le plan proprement musical, en étudiant les textures orchestrales caractéristiques de l'esthétique de Schreker.

Created in 1918, Franz Schreker's opera Die Gezeichneten ("The Stigmatized") has provoked many reactions criticizing the omnipresence of uninhibited and detrimental sexuality, focusing indiscriminately on the subject, the music or the composer himself. The work depicts the tragedy of a young and ugly nobleman, who believed in redemption through love, and sinks into madness after gifting the city of Genoa with an island dedicated to carnal pleasures called "Elysée".

This article seeks to restore the place of the operatic material itself: paradoxically reduced to the anecdotal in its contemporary reception, the work nevertheless deploys powerful mechanisms that, illustrated by the mise en abyme of the Genovese arriving on the island, which has a powerful impact on the spectators. Our study will uncover the means deployed by the opera to impose to its intro-digetic spectator - and by extension the audience of the work - a sensation of stasis that proves to be profoundly erotic. A first section, devoted to the aesthetics of the living painting of the Third Act, analyses the different modalities of visual subjugation to which the Genvese are subjected. In a second section this analysis of erotic stasis will continue but on the musical level, studying the orchestral textures characteristic of Schreker's aesthetic.

\section{INDEX}

Mots-clés : Schreker, Die Gezeichneten, Klang, agentivité, érotisme, tableau vivant, stase Keywords : Schreker, Die Gezeichneten, Klang, agency, eroticism, living painting, stasis 


\section{AUTEUR}

\section{AURORE FLAMION}

Après avoir intégré l'École normale supérieure de Lyon en 2016, Aurore Flamion y poursuit des études de musicologie, ainsi qu'à l'Université Lumière Lyon 2 où elle a rédigé un mémoire consacré aux Stigmatisés de Schreker, sous la direction d’Emmanuel Reibel (« Les Stigmatisés de Schreker. Entre décadence et dégénérescence, généalogie d'un récit idéologique »). Enseignante vacataire à Lyon 2, elle travaille également en tant que rédactrice pour l'Orchestre Victor Hugo Franche-Comté. 